\title{
Applicability of Three Alternative Instruments for Food Authenticity Analysis: GMO Identification
}

\author{
A. Burrell, C. Foy, and M. Burns \\ Molecular and Cell Biology, LGC, Queens Road, Teddington, Middlesex TW11 OLY, UK \\ Correspondence should be addressed to M. Burns, malcolm.burns@lgc.co.uk
}

Received 6 July 2010; Revised 22 November 2010; Accepted 19 December 2010

Academic Editor: Yves Bertheau

Copyright () 2011 A. Burrell et al. This is an open access article distributed under the Creative Commons Attribution License, which permits unrestricted use, distribution, and reproduction in any medium, provided the original work is properly cited.

Ensuring foods are correctly labelled for ingredients derived from genetically modified organisms (GMOs) is an issue facing manufacturers, retailers, and enforcement agencies. DNA approaches for the determination of food authenticitys often use the polymerase chain reaction (PCR), and PCR products can be detected using capillary or gel electrophoresis. This study examines the fitness for purpose of the application of three laboratory electrophoresis instruments (Agilent Bioanalyzer 2100, Lab901 TapeStation, and Shimadzu MCE-202 MultiNA) for the detection of GMOs using PCR based on a previously validated protocol. Whilst minor differences in the performance characteristics of bias and precision were observed, all three instruments demonstrated their applicability in using this protocol for screening of GMO ingredients.

\section{Introduction}

The global food industry faces various challenges in terms of providing confidence in the authenticity of foods. Ensuring foods are correctly labelled for presence and levels of specific ingredients, occurrence of GMOs, and preventing expensive food ingredients being fraudulently replaced with inferior ones, are some of the many issues facing UK industry, Trading Standards, and Public Analysts.

DNA approaches for the determination of food authenticity are becoming increasingly common and easy to implement. These DNA approaches complement results from a number of other analytical approaches (e.g., proteins, chemical analysis, isotope ratios, nuclear magnetic resonance spectroscopy, and Infrared spectroscopy) and are also applicable in instances where protein assays are not considered fit for purpose, for example, when analysing highly processed foods [1]. Use of end-point polymerase chain reaction (PCR) is an effective, cost-efficient DNA approach for determination of food authenticity [2].

Since the digitalisation of electrophoresis, the Agilent Bioanalyzer 2100 has become a well characterised and established instrument, commonly used within the food analysis sector by UK Public Analysts (PAs) who act on behalf of the local government for enforcement and legislative purposes. Many DNA detection and screening protocols that utilise end-point PCR and have been applied on the Agilent Bioanalyzer 2100 have been validated and endorsed by the UK Food Standards Agency (FSA). The Agilent Bioanalyzer 2100 has been shown to be useful as a screening approach in a number of food authenticity studies [3] and can offer an alternative approach compared to instruments using the "gold standard" of real-time PCR, that can be prohibitively expensive to purchase in terms of initial financial outlay and costs associated with maintenance and expertise.

A multiplex PCR screening protocol for the qualitative detection of DNA from GMOs has been characterised extensively [4]. This protocol was tested across ten independent laboratories as part of a blind interlaboratory trial and performance characteristics, including precision, specificity, sensitivity, and associated false positive and negative rates evaluated. The fitness for purpose of this protocol was validated and demonstrated using the Agilent Bioanalyzer [5].

Recently, several alternative electrophoresis instruments have emerged onto the market, reporting very similar or improved performance, increased range of applications, or greater cost efficiency compared to the Agilent Bioanalyzer 
$2100[6,7]$. Examples of alternative electrophoresis instruments include the range of Applied Biosystems capillary electrophoresis instruments (e.g., the ABI PRISM 3100 Genetic Analyzer, the Beckman Coulter GenomeLab GeXP Genetic Analysis System, the Caliper LabChipGXII, the Lab901 TapeStation, the QIAGEN QIAxcel System, and the Shimadzu MCE-202 MultiNA). The applicability of using the multiplex PCR screening protocol for detection of GMO ingredients [4] on these alternative electrophoresis instruments has not previously been reported or characterised. Demonstrating the application of a protocol for the detection of GMO ingredients across a range of electrophoresis machines would increase the scope and potential choice of instruments available for the food authenticity sector.

The aim of this study was to perform a comparative analysis of three electrophoresis instruments, with respect to their fitness for purpose in using a previously validated multiplex PCR screening protocol for the qualitative detection of DNA from GMOs. The Agilent Bioanalyzer 2100, Lab901 TapeStation, and the Shimadzu MCE-202 MultiNA were selected as a representative cross section of electrophoresis instruments available. DNA profiles from these three instruments were compared objectively, and estimation of the performance characteristics of bias and precision made. The applicability of all three instruments in successfully applying the previously validated protocol for screening of GMO ingredients was demonstrated, and these may provide alternative choices for those laboratories involved in food analysis which do not always have access to real-time PCR.

\section{Materials and Methods}

2.1. "GMO Multiplex Screen" Protocol. The FSA protocol for genetically modified organism (GMO) detection "GMO multiplex screen protocol: Standard operating procedure for the simultaneous detection of general DNA targets associated with the presence of genetically modified soya and maize" [4] was used, hereafter referred to as the "GMO multiplex screen" protocol. Standard end-point PCR was conducted following the "GMO multiplex screen" protocol, which was designed to be a simple, cost-effective protocol for screening food ingredients, animal feed, and some processed foods. It is a qualitative multiplex end-point PCR approach allowing simultaneous detection of wild-type and GM soya and maize and cauliflower mosaic virus (CaMV). The protocol allowed DNA amplicons representing Zein (maize reference gene), Lectin (soya reference gene), cauliflower mosaic virus (CaMV viral DNA), and NOS terminator/CaMV 35s promoter (control genes indicative of genetic modification) to be generated. The assay included the CaMV target, that does not crossreact with the $35 \mathrm{~S}$ gene, to allow samples that contained GMO ingredients to be differentiated from samples that contained material from cruciferous plants that had been naturally infected by the CaMV virus [4]. The "GMO multiplex screen" protocol, optimised and validated for use on the Agilent Bioanalyzer 2100, has not been extensively tested on alternative electrophoresis instruments.
2.2. DNA Samples. A set of test samples and positive controls were provided as part of the validation of "GMO multiplex screen" trial for Public Analysts during 2008. DNA was extracted using a modified CTAB method [8], resuspended in water, and stored at $-20^{\circ} \mathrm{C}$.

Two control materials and six blind test samples were evaluated as part of the current study. The test samples consisted of maize flour (contained $0.1 \%$ Bt11 maize); spice mix (CaMV and trace levels of maize); textured vegetable protein (3\% Roundup Ready soya and trace levels of maize); maize flour (1\% Bt11 maize); maize polenta (no GM); and animal feed ( $3 \%$ Roundup Ready soya and trace levels of maize). The first positive control consisted of $0.5 \%$ GM soya and CaMV DNA. The second positive control contained approximately $1 \mathrm{ng}$ of DNA extracted from $5 \% \mathrm{w} / \mathrm{w}$ Roundup Ready soya certified reference material [9], $1 \mathrm{ng}$ of DNA from a $5 \%$ w/w NK603 maize certified reference material [10], and an authenticated, but unspecified, amount of DNA from CaMV. This second positive control produced all bands (Zein, Lectin, CaMV, and the NOS terminator/CaMV 35s promoter complex) when used in end-point PCR at a final amount of $20 \mathrm{ng}$ DNA per reaction [4]. The DNA concentration in each sample was quantified and diluted to final amounts corresponding to approximately 20 or $100 \mathrm{ng}$ per reaction for end-point PCR. Sterile nuclease-free water was used as an appropriate negative control for extraction purposes.

2.3. PCR. The PCR consisted of a multiplex assay containing five assays detecting CaMV, CaMV 35s promoter, NOS terminator, Lectin, and Zein amplicons. Primer sequences were as detailed in the original protocol [4]. Each reaction contained either $20 \mathrm{ng}$ or $100 \mathrm{ng}$ DNA, 1x QIAGEN Multiplex PCR Mastermix, primer master mix solution at $100 \mathrm{nM}$ per primer, and the remaining reaction volume was made up to $15 \mu \mathrm{L}$ with sterile water, as per the original protocol [4]. Cycling conditions were as follows: $95^{\circ} \mathrm{C}$ for $15 \mathrm{mins}$, 40 cycles of $95^{\circ} \mathrm{C}, 25 \mathrm{sec} ; 62^{\circ} \mathrm{C}, 30 \mathrm{sec} ; 72^{\circ} \mathrm{C}, 45 \mathrm{sec}$; then $7 \mathrm{~min}$ at $72^{\circ} \mathrm{C}$ and held at $4^{\circ} \mathrm{C}$. The PCR was performed on an MJ Research PTC-225 Thermal Cycler (GRI LabCare, Rayne, UK). Samples were analysed based on the proprietary software associated with each of the three electrophoresis instruments, using the most appropriate kit available for detection of PCR products between $50 \mathrm{bp}$ and $200 \mathrm{bp}$.

\subsection{Instruments}

2.4.1. Agilent Bioanalyzer 2100. The Agilent 2100 Bioanalyzer (Agilent Technologies, Santa Clara, California) is a capillary electrophoresis-based system that can analyse DNA, RNA, and proteins. It has a sample throughput of 12 samples per run, and results are generated in approximately 30 minutes [11]. Capillary electrophoresis is used to separate DNA fragments based upon size and charge. PCR products are sized accurately and quantified by alignment with DNA fragments from a ladder.

PCR products were analysed using the DNA 1000 LabChips on the Agilent Bioanalyzer 2100. All chips were 
run as per the manufacturer's instructions provided with the DNA 1000 LabChip kit [11]. Fresh gel-dye matrix was prepared with $20 \mu \mathrm{l}$ of the dye concentrate and $400 \mu \mathrm{l}$ of the gel matrix; the mixture was filtered through a spin filter and used in the LabChip. $5 \mu \mathrm{l}$ of the DNA marker reagent was added to each sample well, $1 \mu \mathrm{l}$ DNA ladder reagent was added to the assigned ladder well, and $1 \mu \mathrm{l}$ sample was added to the corresponding sample wells. The LabChip was vortexed and run on the Agilent Bioanalyzer 2100 using the Expert 2100 software.

2.4.2. Lab901 TapeStation. The Lab901 TapeStation (Lab901 Limited, Loanhead, United Kingdom) is an automated gel electrophoresis system and can facilitate the analysis of protein, RNA, and DNA. The ScreenTape consist of 16 individual separation channels preloaded with reagents. The instrument used in this study utilised the reusable ScreenTape system for the analysis of 16 samples simultaneously within one run, but depending on the sample format between 1 and 96 samples can be analysed on the instrument.

PCR products were analysed using the DNA 800 Tape kit on the Lab901 TapeStation. All tapes were run as per the manufacturer's instructions provided with the DNA 800 Tapes [12]. Samples were loaded into $0.2 \mathrm{ml} \times 8$ connected PCR tubes; $5 \mu \mathrm{l}$ neat DNA ladder was added to the first tube, then $4 \mu \mathrm{l}$ Loading Buffer and $1 \mu \mathrm{l}$ of sample were added to each subsequent tube. Lids were placed on the $0.2 \mathrm{ml}$ tubes, and the samples were briefly vortexed and centrifuged. The tubes, recommended tips, and Tape were placed in the Lab901 TapeStation and ran using the TapeStation Controller Software GeneTools.

2.4.3. Shimadzu MCE-202 MultiNA. The Shimadzu MCE202 MultiNA (Shimadzu Corporation, Kyoto, Japan) uses microchip technology to facilitate capillary electrophoresis for nucleic acid analysis. This instrument demonstrated a high sample throughput and can use 8 or 12 strip tubes or 96 well plate formats. PCR products were analysed using the DNA 500 kit on the Shimadzu MCE-202 MultiNA. All sample runs were performed by the manufacturer. Samples were run with the reagents from the DNA 500 kit: separation buffer, DNA marker reagent, and 25 bp DNA ladder. The samples were placed into the MultiNA instrument alongside the reagents. The samples and reagents were mixed automatically on-chip and ran using the MultiNA Control and MultiNA Viewer software [13].

2.4.4. Experimental Design. Two replicate runs per instrument were conducted, and two positive controls were implemented within each chip/tape per replicate run, in order to estimate the within and between run repeatability associated with PCR amplicon sizes. Due to space constraints on the chips/tapes, the complete set of test samples had to be divided across two chips/tapes per run for both the Agilent Bioanalyzer 2100 and the Lab901 TapeStation instruments. However, all samples could be accommodated within one Shimadzu MultiNA chip, so each of the duplicate runs on this instrument consisted of just one chip. Consequently, the Agilent Bioanalyzer 2100 and Lab901 TapeStation experiments were represented by eight replicates of the positive control (four per duplicate run), and the Shimadzu MultiNA experiment was represented by four replicates of the positive control (two per duplicate run).

\section{Results and Discussion}

3.1. "GMO Multiplex Screen" Protocol. The "GMO multiplex screen" protocol is a qualitative multiplex end-point PCR approach that allows simultaneous detection of wild-type and GM soya and maize and Cauliflower Mosaic Virus (CaMV) in food samples. The protocol has been evaluated extensively on the Agilent Bioanalyzer [5] but has not been tested comprehensively on other electrophoresis instruments.

The "GMO multiplex screen" PCR contains five primer pairs that produce amplicons from the following DNA targets: the maize zein gene, soya lectin gene, CaMV, NOS terminator, and CaMV 35s promoter. Primer sets for the zein, lectin, NOS terminator, and CaMV $35 \mathrm{~S}$ promoter were selected from the British Standard (BS EN ISO) 21569:2005 and previously published papers. The CaMV primer pair was designed as part of the original study [5] to only amplify DNA from the naturally occurring cauliflower mosaic virus, and not to amplify DNA from the CaMV $35 \mathrm{~S}$ promoter sequence used for genetic transformation. Specificity was tested theoretically (based on available database information) and confirmed in the laboratory against DNA extracted from a range of cereals and common food ingredients with no reported false positives [5].

As part of the original study [5], all primers pairs were tested against dilutions from appropriate certified reference materials containing GM ingredients. Estimated limits of detection for the lectin and zein targets, and the $35 \mathrm{~S}$ and NOS targets, corresponded approximately to less than 20 and less than 10 genome equivalents, respectively. Previously the "GMO multiplex screen" has been applied to a range of textured vegetable protein and animal feed samples from GeMMA proficiency test rounds and commercially available animal feed samples, containing between $0.5 \%$ and $3 \%$ $\mathrm{w} / \mathrm{w}$ GM maize and soya ingredients. The screening assay successfully detected GM ingredients in all of the positive test samples analysed, and no false negatives were reported.

As part of this original study, the "GMO multiplex screen" protocol was evaluated further by a blind interlaboratory trial involving ten independent laboratories. Two positive control materials and six blind test samples were evaluated as part of the trial. All samples were run at two dilution levels (corresponding to approximately 20 and $100 \mathrm{ng}$ of DNA per reaction) in duplicate using PCR and visualised on the Agilent Bioanalyzer. Negative controls were included as no template controls (water replacing DNA in the PCR wells) and extraction blanks. The trial organisers did not report any confirmed false positive responses from the ten laboratories as part of this trial, further confirming the specificity of the assay. As part of this trial, 100\% (nine out of nine) of the results obtained showed that $0.5 \% \mathrm{w} / \mathrm{w}$ GM soya 
TABle 1: Amplicon sizes associated with the "GMO Multiplex Screen" protocol. Amplicons sizes are shown in bp. "Theoretical size (bp)" refers to the calculated number of base pairs in the amplicon based on available DNA sequence information. The final three columns refer to the average bp sizes of the amplicons as determined by the Agilent Bioanalyzer 2100, Lab901 TapeStation, and Shimadzu MultiNA, for the positive control used in this study. Note that the NOS terminator and CaMV 35s promoter PCR products were seen as one peak on all three instruments.

\begin{tabular}{lcccc}
\hline Primer set & $\begin{array}{c}\text { Theoretical size } \\
(\mathrm{bp})\end{array}$ & $\begin{array}{c}\text { Agilent Bioanalyzer 2100 sizes } \\
(\mathrm{bp})\end{array}$ & $\begin{array}{c}\text { Lab901 TapeStation sizes } \\
(\mathrm{bp})\end{array}$ & $\begin{array}{c}\text { Shimadzu MultiNA sizes } \\
(\mathrm{bp})\end{array}$ \\
\hline Zein & 68 & 80 & 71 & 77 \\
Lectin & 80 & 90 & 81 & 86 \\
CaMV & 104 & 108 & 104 & 108 \\
NOS terminator & 118 & 131 & 120 & 127 \\
CaMV 35S promoter & 123 & & & \\
\hline
\end{tabular}

was detected repeatably in the first positive control. Eight of nine laboratories that reported results were able to detect the $0.1 \% \mathrm{w} / \mathrm{w}$ Bt11 maize flour test sample. This confirmed the sensitivity of the assay and suggested that the limit of detection was between $0.5 \%$ and $0.1 \% \mathrm{w} / \mathrm{w}$ GM soya/maize of a particular ingredient. The original study stated that the assay could detect down to $0.1 \%$ (w/w) levels of GMO [4].

The study concluded that the GMO screening assay was beneficial in terms of providing a cost-effective screening method for GM for those laboratories that could not afford to purchase and maintain high-throughput real-time PCR capabilities. The "GMO multiplex screen" protocol can be used as a preliminary screening approach to investigate samples that may contain GM DNA.

3.2. Generation of DNA Profiles. As part of the current study, the series of test samples and positive controls were evaluated across the three instruments of the Agilent Bioanalyzer, the Lab901 TapeStation, and the Shimadzu MultiNA. All instruments identified the presence of the correct bands in the positive controls, and there was no evidence of contamination in the negative controls. Table 1 shows that all three instruments were able to correctly identify individual PCR amplicons of Zein, Lectin, CaMV, and an NOS terminator/CaMV 35S promoter complex, which arose from the profile of the positive control. Within an instrument, all amplicons were clearly distinguishable from each other. Whilst the profile from the positive control was consistent across all three instruments, the estimated size of the individual amplicons was different between instruments.

All three instruments detected the expected amplicons for the set of six test samples when using the recommended $20 \mathrm{ng}$ and $100 \mathrm{ng}$ DNA per reaction from the protocol (data not shown). This result was consistent between all three instruments and in line with previous results [5]. Therefore, all three instruments could be used to detect and differentiate between samples containing wild-type maize, GM maize, wild-type soya, GM soya, and the presence of CaMV viral DNA.

The positive controls and test samples generally showed that the lower DNA template concentration (20 ng) gave more reliable results for the presence/absence of amplicons compared to the higher concentration (100 ng), as observed from previous trials of the protocol on the Agilent Bioanalyzer 2100 [5]. The NOS terminator and CaMV 35s promoter amplicons were seen as one peak for all instruments, consistent with the original protocol [4]. Further optimisation of the GMO screening protocol [4] could be aimed at determining if it is possible to resolve these two closely related peaks, or redesigning of the primers to give different amplicon sizes.

Whilst the individual sizes of the amplicons within the profile for the positive control differed between instruments, a consistent DNA profile for the positive control and all test samples was given across the three instruments. Each of the instruments could therefore be used to detect and differentiate between samples that contain normal and GM soya, and normal and GM maize, as well as identify the presence of CaMV viral DNA that may be naturally occurring with plant and soil samples.

3.3. Evaluation of Performance Characteristics. Having obtained the correct amplicon profiles across all three instruments demonstrates the applicability of the three instruments for using the "GMO multiplex screen" protocol, previously validated on the Agilent Bioanalyzer. A subset of the data, consisting of the replicate positive controls, was then used to further characterise the performance characteristics of precision (repeatability) and bias (trueness) associated with amplicon sizes across the three instruments. This data was derived from two replicate runs per instrument, where each chip/tape within a replicate run contained at least two positive controls. Because of the different sample throughput associated with the three instruments for analysing the test samples, only a limited number of reaction wells could be used for the positive controls to estimate within and between run variability. For the Bioanalyzer and TapeStation instruments, four replicate positive controls were tested per run (eight replicates in total), and for the MultiNA, two replicate positive controls were tested per run (four in total). For each of the positive control replicates, the estimated size of the four amplicons (Zein, Lectin, CaMV, and the combined NOS terminator/CaMV 35S promoter complex) was measured. This level of replication, which is limited by the throughput of the instruments, provided a first insight into evaluation of the performance characteristics or trueness and precision to allow a comparison between the instruments. 


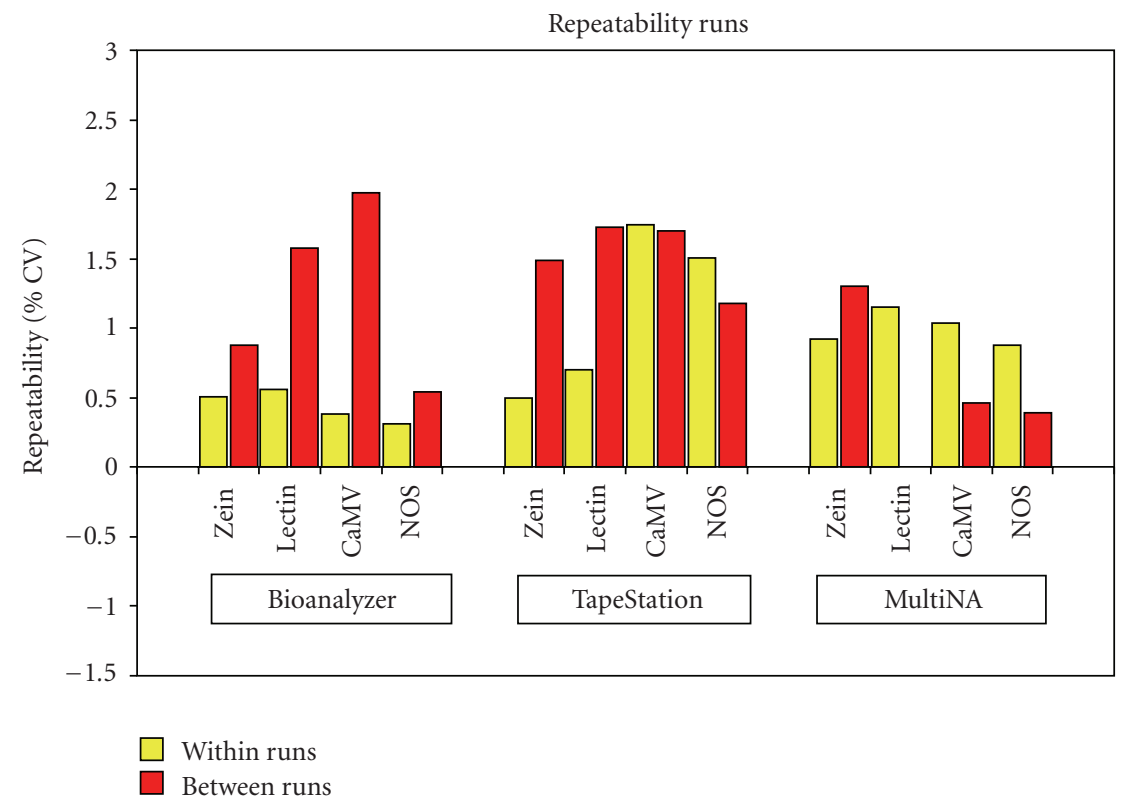

FIGURE 1: Repeatability (\%CV) associated with the positive control. Figure 1 shows the precision, expressed as the percentage coefficient of variation, for each of the four amplicons in the positive controls, per instrument. The values are based on the repeatability both within and between the two replicate runs for amplicon size. Note: the NOS data consists of the combined NOS terminator/CaMV $35 S$ promoter complex.

3.4. Precision (Repeatability). Repeatability was calculated based on the measurement response of estimated basepairs (bp) size of each of the amplicons. Repeatability was expressed as the percentage coefficient of variation $(\% \mathrm{CV})$. Figure 1 shows the repeatability within and between the two replicate runs per instrument, based on amplicon size.

The Agilent Bioanalyzer 2100 demonstrated the least within-run variability for estimated amplicon size for all four amplicons in the positive controls. However, whilst the between-run repeatability for the Zein and NOS amplicons was relatively good, the CaMV amplicon had the largest between-run variability associated with it across all three instruments.

The Lab901 TapeStation had a larger CV associated with estimated amplicon size overall, and this was particularly evident with the CaMV and NOS terminator/CaMV 35s promoter complex amplicons.

The Shimadzu MultiNa appeared to exhibit relatively large within-run variation for the Zein and Lectin amplicons compared to the other two instruments. However, the between-run variability was very good for the Lectin, CaMV and the combined NOS terminator/CaMV 35s promoter complex. In Figure 1, the absence of the repeatability estimate for the between-run repeatability associated with the Lectin amplicon does not represent missing data but indicates that there was no variability between the two runs in terms of amplicon size (both runs gave an average estimated amplicon size of $87 \mathrm{bp}$ ) resulting in a CV of $0 \%$.

Whilst there were minor differences associated with the repeatability estimates of the amplicon sizes between the three instruments, the CV associated with any amplicon never exceeded $1.75 \%$ and $1.98 \%$ for the within- and between-run repeatability estimates, respectively. Therefore, all three instruments showed good repeatability of the amplicon sizing within and between chip/tapes and runs in absolute terms. This further demonstrated the fitness for purpose of all three instruments in using the "GMO Multiplex Screen" protocol.

3.5. Bias (Trueness). Table 1 shows the amplicon sizes associated with the positive control. The theoretical size refers to the theoretical number of base pairs associated with each amplicon based on available DNA sequence information. This theoretical amplicon size was used as a measure of the bias (trueness) associated with the results produced by all three instruments and was calculated across all replicate runs within the experiment (Figure 2).

Overall, the TapeStation gave estimated amplicon sizes very close to the theoretical sizes, as shown in Table 1 and Figure 2. Any observable differences were well within the sizing specification associated with the instrument.

The MultiNA and Bioanalyzer 2100 overestimated the theoretical sizes slightly. Overall, the MultiNA demonstrated less bias than the Bioanalyzer 2100. Whilst the MultiNA demonstrated some positive bias, the estimated distances between the four amplicons in the positive control compared well with the distances separating the four amplicons based on theoretical size. The Bioanalyzer 2100 demonstrated the most bias compared with theoretical amplicon sizes, and the estimated distances between the four amplicons did not compare as well with the distances separating the four amplicons based on theoretical size.

However, in absolute terms, the differences in bias and amplicon sizing were very small and did not affect 


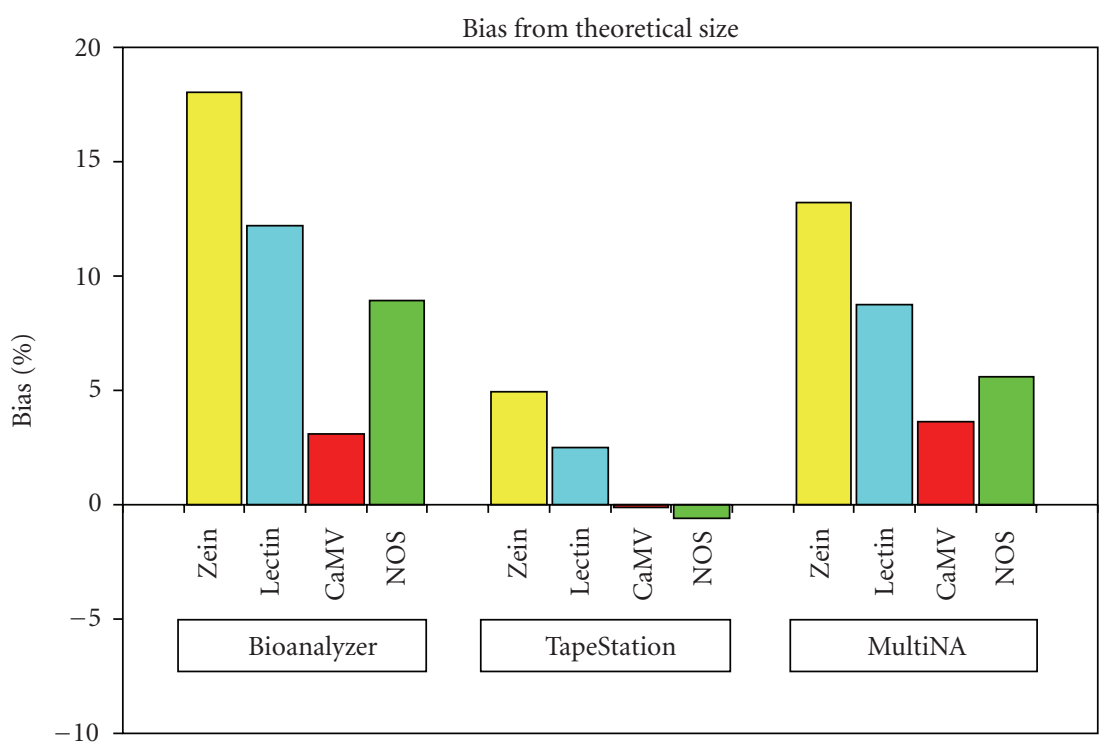

FIGURE 2: Bias associated with the amplicons from the positive controls. Figure shows the bias (trueness) associated with the amplicons from the positive controls, across the three instruments of the Bioanalyzer 2100, TapeStation, and MultiNA. The bias is based on the difference between the observed amplicon size per instrument, compared with the theoretical amplicon size based on the sequence information. NOS represents the combined NOS terminator/CaMV 35s promoter amplicon. Bias is calculated on the measurement response of base pairs (bp).

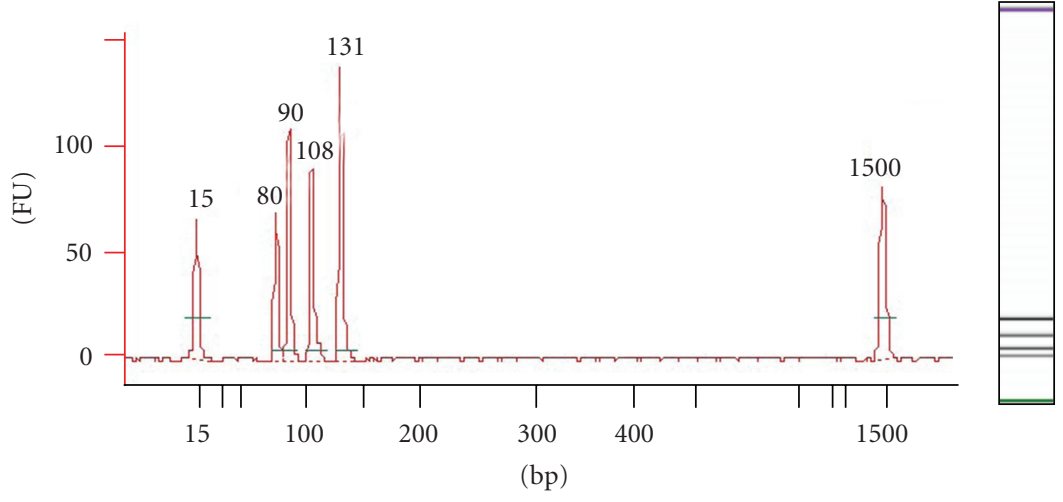

Figure 3: Electropherogram and "gel-like" image of positive control on the Agilent Bioanalyzer 2100. The $x$-axis on the electropherogram represents amplicon size (bp), whist the $y$-axis represents the measurement response of fluorescence units (FUs). Amplicons of interest in order of size (bp) are lower marker (15), Zein (80), Lectin (90), CaMV (108) and combined CaMV 35s promoter/NOS terminator (131) amplicons, and the upper marker (1500).

the interpretation of the DNA profile generated by the samples. All three instruments were easy to use to correctly identify the positive controls and test samples.

\subsection{Review of Instruments}

3.6.1. Agilent Bioanalyzer 2100. Agilent Technologies state the Bioanalyzer 2100's advantages to be ease-of-use, speed of analysis, low sample volumes, and high reproducibility [9]. This instrument showed the least variability when repeatedly determining amplicon size, correctly identified the amplicons, and was able to quantify their concentration. This instrument exhibited some small bias in overestimating amplicon sizes compared to theoretical size. Figure 3 is an electropherogram and a "gel-like" view of a positive control sample; both clearly distinguish the amplicons.

3.6.2. Lab901 TapeStation. Lab901 states the TapeStation's advantages to be a simple, reliable, and fast DNA electrophoresis [12]. This instrument showed the least bias results of all instruments when comparing estimated amplicon sizes to theoretical sizes and correctly identified the amplicons despite being performed using a protocol optimised for the Bioanalyzer 2100. The instrument demonstrated some variability in terms of amplicon size estimation, but this was always below a CV of $1.87 \%$. However, at the stage this study was conducted, the instrument could not accurately quantify the DNA concentration arising from 


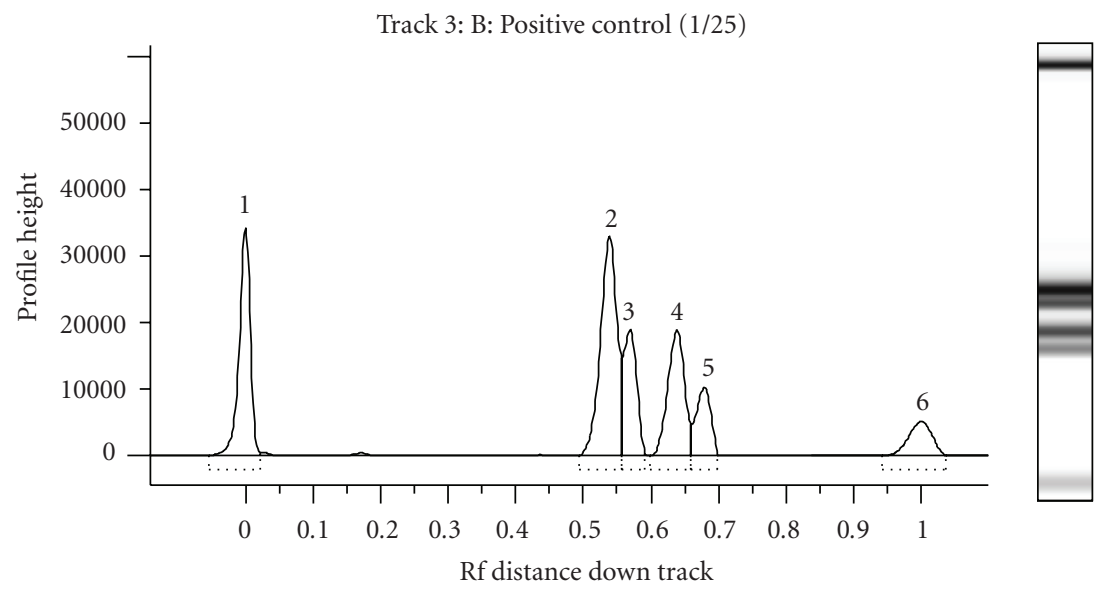

Figure 4: Electropherogram and "gel-like" image of positive control on the Lab901 TapeStation. The $x$-axis on the electropherogram represents the distance the amplicon has migrated, whist the $y$-axis represents the measurement response in fluorescence units. Amplicons of interest in order of size are (1) lower marker, (2) Zein 77 bp, (3) Lectin 86 bp, (4) CaMV 104 bp and (5) combined CaMV 35s promoter/NOS terminator $120 \mathrm{bp}$ amplicons, and (6) upper marker.

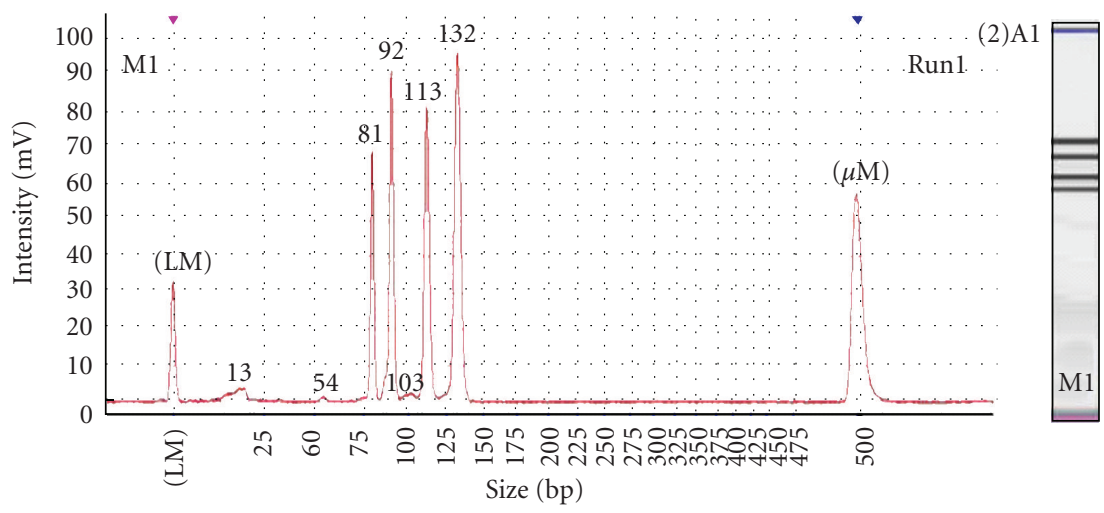

FIgURE 5: Electropherogram and "gel-like" image of positive control on the Shimadzu MultiNA. The $x$-axis on the electropherogram represents amplicon size (bp), whist the $y$-axis represents the measurement response of fluorescence units. Amplicons of interest in order of size (bp) are lower marker, Zein (81), Lectin (92), CaMV (113) and combined CaMV 35s promoter/NOS terminator (132) amplicons, and the upper marker.

each amplicon. Potential advances in the associated TapeStation Screenplex software may provide opportunities for automation and data standardisation in terms of operatorindependent multiple amplicon analysis. Figure 4 is an electropherogram and a "gel-like" view of a positive control; both clearly distinguish the amplicons.

3.6.3. Shimadzu MCE-202 MultiNA. The manufacturer states the MCE-202 MultiNA's advantages to be highspeed and highly reproducible sample size confirmation on a reusable microchip [13]. This instrument correctly identified and was able to quantify the concentrations of the amplicons, despite being performed using a protocol validated on the Bioanalyzer 2100. The instrument exhibits a very high sample throughput, allowing up to 96 samples to be analysed on one reusable chip, making it a very cost-efficient instrument. There was some small bias in overestimating the amplicon sizes compared to the theoretical amplicon sizes. Figure 5 is an electrophoregram and a "gel-like" view of a positive control; both clearly distinguish the amplicons.

A comparative analysis of the application of the three alternative electrophoresis instruments of the Agilent Bioanalyzer 2100, Lab901 TapeStation, and the Shimadzu MCE202 MultiNA was conducted as part of the current study, showing the fitness for purpose of the "GMO multiplex screen" protocol across all three instruments. These three instruments were chosen as a representative cross section of some of the commercially available electrophoresis systems currently on the market. Other electrophoresis instruments are available, and based on the results presented here, it is likely that the "GMO multiplex screen" protocol will give good results when applied on these additional instruments.

\section{Conclusion}

This "GMO multiplex screen" protocol for GMO detection [4] has been extensively trialled and validated on the Agilent 
Bioanalyzer as part of a previous study [5], and it was stated that this approach may provide an alternative route for detecting GMO ingredients for those laboratories that do not have access to real-time PCR. Whilst the protocol had been validated on the Agilent Bioanalyzer, its application had not been reported or extensively tested on other alternative electrophoresis instruments.

Overall, all three instruments performed well when applying the "GMO multiplex screen" protocol and offer suitable alternative instruments on which to use the protocol. Based on a limited data set, the Agilent Bioanalyzer 2100 appeared the most precise when estimating amplicon size, both within and between runs, when using the "GMO multiplex screen" protocol. The Lab901 TapeStation exhibited the least bias from the theoretical size of the amplicons using samples evaluated in this study and was the only technology to correctly size the amplicon in comparison to their theoretical sizes; both the Bioanalyzer 2100 and MultiNA instruments tended to overestimate amplicon sizes slightly compared to the theoretical size. The MultiNA instrument demonstrated excellent repeatability of most amplicons between runs. However, the observed bias and repeatability never exceeded $18 \%$ and $2 \%$, respectively. Whilst there were minor deviations in relation to bias and precision estimates, all three instruments were able to correctly detect the presence or absence of the different amplicons and generated the correct characteristic DNA profiles used in this study for a range of test samples and the positive controls. In this respect, all three instruments performed fit for purpose in relation to the application of the "GMO multiplex screen" protocol, and as alternative instruments, they provide greater scope for analysis of food authenticity.

The fitness for purpose of a prevalidated "GMO multiplex screen" protocol was demonstrated as part of a comparative analysis using just three electrophoresis instruments. The authors emphasise that alternative electrophoresis instruments are commercially available that were not evaluated as part of this study. Based on the evidence presented here, it is likely that the application of the "GMO multiplex screen" protocol would give good results across a range of alternative instruments.

\section{Acknowledgments}

The authors thank Steve Garrett (Campden BRI, UK) for the useful advice and encouragement in the production of this paper. The work described in this paper was funded by the UK National Measurement System as part of the "Government Chemist 2008-2011 Programme."

\section{References}

[1] M. Burns, R. Sanders, and A. Burrell, "Strawberry and raspberry fruit differentiation using the Agilent CE 2100 Bioanalyzer. Agilent Technologies application note," 2008, https://cp.chem.agilent.com/Library/applications/5990-3327 EN.pdf.
[2] Food Standards Agency, "Research and Survey ProgrammesAnnual Report 2007,” FSA/1194/0907, 2007, http://www .food.gov.uk/multimedia/pdfs/researchreport2007.pdf.

[3] Food Standards Agency, "Research and Survey ProgrammesAnnual Report 2007: Project Q01099, Extending the labon-a-chip PCR-RFLP database for a wider range of commercial fish species," FSA/1194/0907, 2007, http://www .food.gov.uk/multimedia/pdfs/researchreport2007.pdf.

[4] S. Garrett, "Standard operating procedure for the simultaneous detection of general DNA targets associated with the presence of genetically modified soya and maize," Campden BRI, 2008, http://www.foodbase.org.uk//admin tools/reportdocuments/300-1-532_Appendix_F._SOP_GMO_ multiplex_PCR_screen.pdf.

[5] S. Garrett, J. Dooley, M.-A. Clarke, and H. Brown, "Refinement of GMO screening methods by combining existing multiplex PCR approaches with lab-on-a-chip capillary electrophoresis endpoint detection," Final Technical Report-Food Standard Agency Project G03022, Campden BRI,http//wwwfoodbase.org.uk//admintools/reportdocuments 300-1-525_G03022_FSA_GMO_Project_Final_Report.pdf.

[6] http://www.lab901.net/images/pdfs/Lab901_D800_Optimised .pdf.

[7] http://www.shimadzu-biotech.net/pages/products/2/multina .php.

[8] M. Burns and H. Valdivia, "A simulation approach to assess the minimal number of real-time PCR replicates for GM quantification," European Food Research and Technology, vol. 227, no. 6, pp. 1721-1727, 2008.

[9] S. Trapmann, P. Catalani, P. Conneely et al., "The Certification of Reference Materials of Dry-mixed Soya Powder with different Mass Fractions of Roundup Ready ${ }^{\mathrm{TM}}$ Soya-IRMM410S," 2002.

[10] Certificate of analysis, ERM-BF415f, https://irmm.jrc.ec .europa.eu/refmat_pdf/ERM-BF415f_cert.pdf.

[11] Agilent Technologies, "Agilent DNA 1000 Kit Guide," Manual Part G2938-90014, 2006, http://www.chem.agilent.com/ Library/usermanuals/Public/G2938-90014_KitGuideDNA1000 Assay_ebook.pdf.

[12] Lab901 ScreenTape® System User Manual_GeneTools Software. ScreenTape® System User Manual-GeneTools® Software. Edition A-,Lab901 Ltd., 2010, http:/www.lab901.net/ images/downloadabledocs2/lmm-016-a-genetools_um.pdf.

[13] Shimadzu, Microchip Electrophoresis system for DNA/RNA, MCE-202 MultiNA Instruction Manual Operating Procedure, 292-28464D, 2008. 

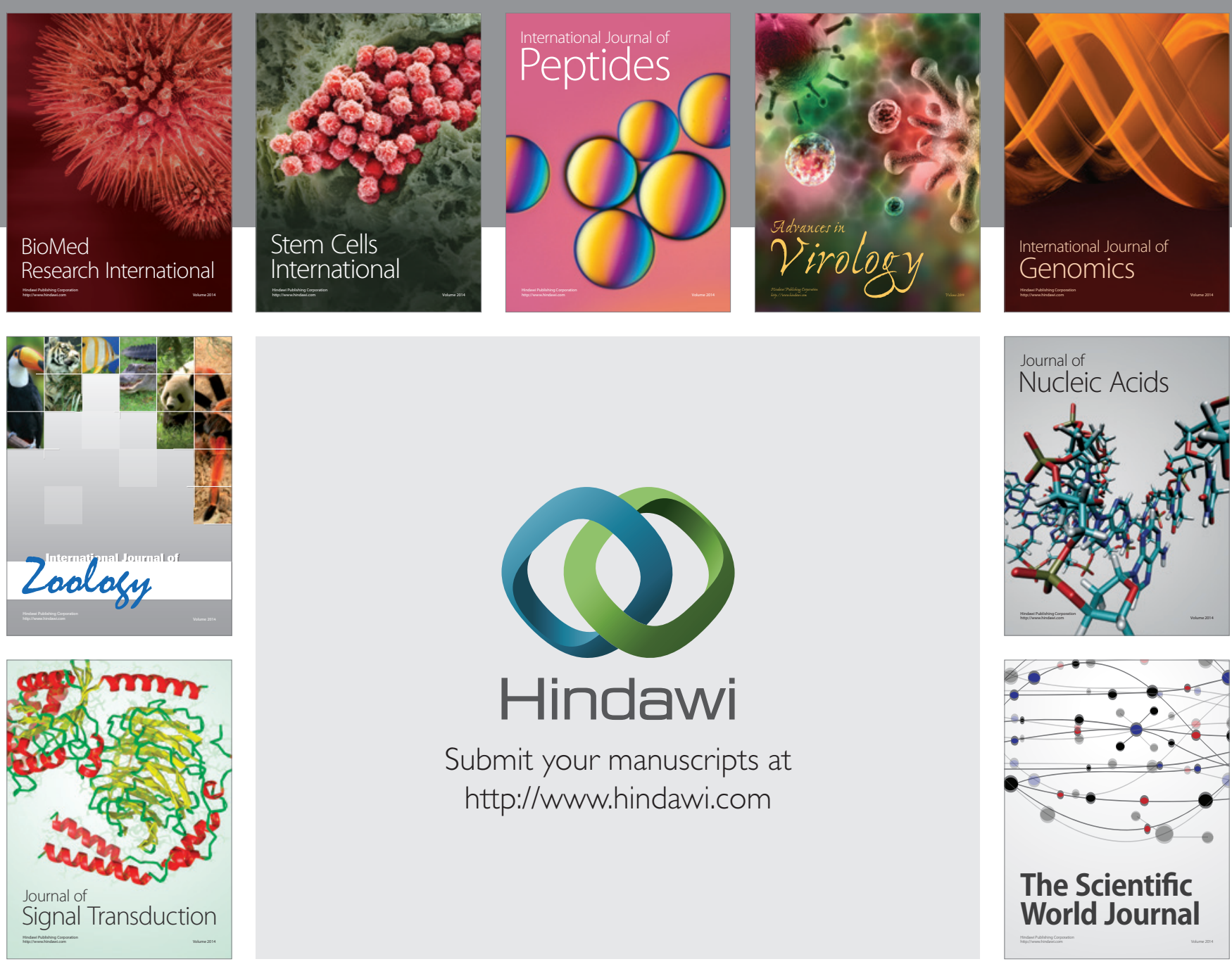

Submit your manuscripts at

http://www.hindawi.com
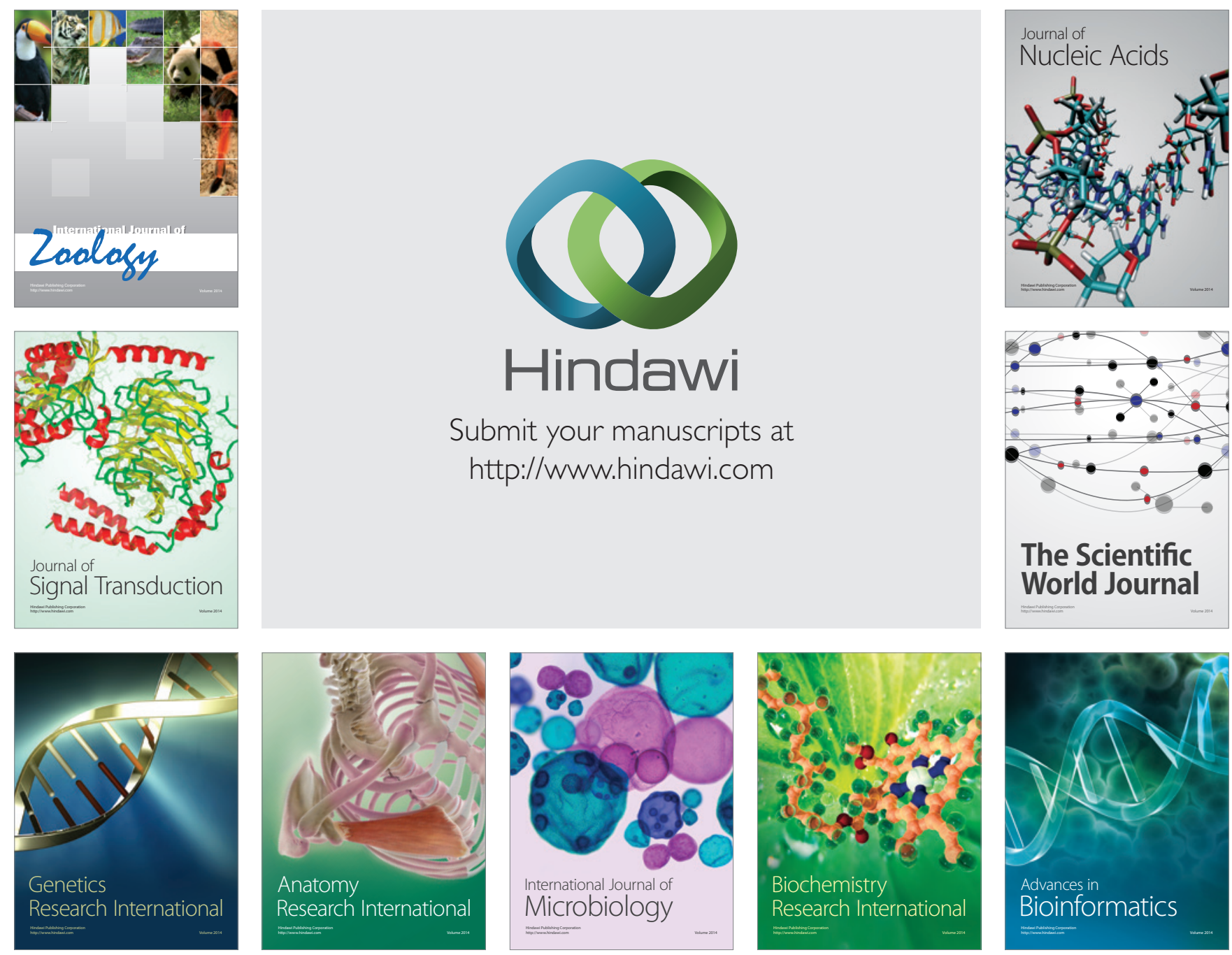

The Scientific World Journal
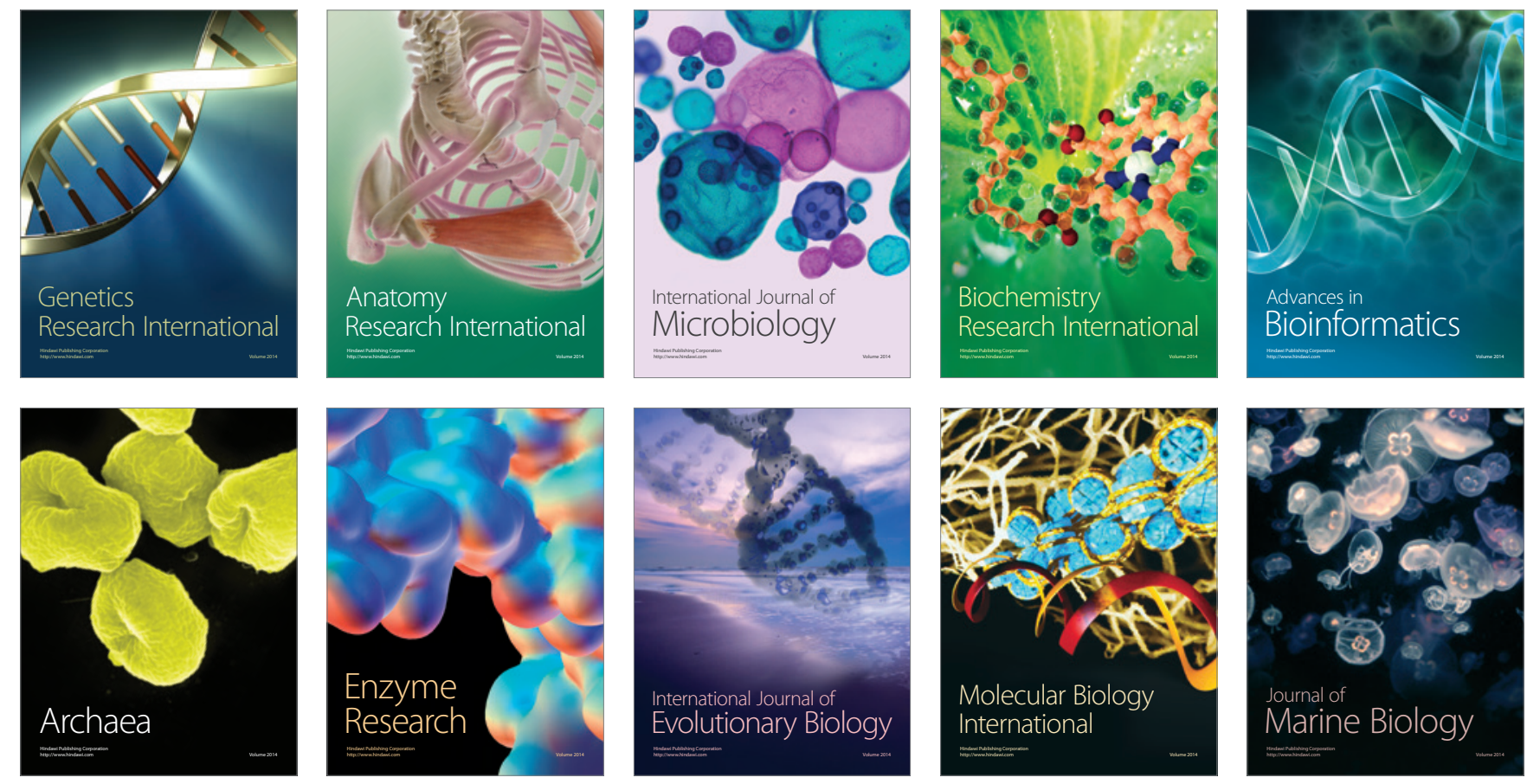